For every subset $W \subset X, \overline{\bar{W}} \leqslant \mathfrak{m}$, and for every $\varphi \in Z$, let $L_{W, \varphi}$ be VOL. XI 1963 FASC. 1 a. subset of $Z$ defined as follows:

$$
f \epsilon L_{W, p} \text { if and only if } f|W=\varphi| W
$$

(i. e. if $f(x)=\varphi(x)$ for every $x \in W$ ).

$F$ is the m-field of subsets of $Z$ generated by all the sets $L_{W, \varphi}$.

Now we are going to prove that $F^{\mathfrak{m}}$ is not wealkly $\mathfrak{m}$-distributive, if $\mathfrak{n} \geqslant \mathfrak{m}^{+}$.

Let

$$
T^{\prime}(x, \eta)=\{f \in Z: f(x)=\eta\},
$$

and let

$$
A_{\eta}=\{T(x, \eta): x \in X\} \text {. }
$$

Every family $A_{\eta}, \eta \in Y, \mathfrak{m}^{+}$-covers $F^{\prime}$ (see [2], p. 139).

It follows from the definition of $Z$ that

$$
\bigcap_{\eta \in X} \bigcup_{x \in X} T(x, \eta)=0,
$$

where the intersection and the union are set-theoretical.

Suppose that there exists a covering $A$ of $F$ which m-refines every $A_{\eta}$. Thus, the field $F$ being m-complete, every element of $A$ is included in the set-theoretical union of elements of $A_{\eta}$, for every $\eta \in \bar{Y}$. Therefore it is empty, by (i). Contradiction.

Consequently, by lemma $2.5, F^{\mathrm{m}}$ is not weakly m-distributive.

\title{
REFERENCES
}

[1] S. Banach et C. Kuratowski, Sur une généralisation du problème de la mesure, Fundamenta Mathematicae 14 (1929), p. 127-131.

[2] R. S. Pierce, Distributivity and the normal completion of Boolean algebras, Pacific Journal of Mathematics 8 (1958), p. $133-140$.

[3] R. Sikorski, Boolean algebras, Berlin-Göttingen-Heidelberg 1960.

Regn par la Rélaction le 8. 8. 196 ?

\section{A FEW PROBLEMS ON BOOLEAN ALGEBRAS}

$\mathrm{BX}$

ROMAN SIKORSKI (WARSAW)

The purpose of this short note is to collect a few problems concerning Boolean algebras which seem to be interesting. Some of them were mentioned in my expository paper [7], others were quoted in my book [9]. Perhaps, the level of difficulty of some of them is rather low. In any case, their solutions will mean a progress in the theory of Boolean algebras.

The first problem concerns the following simple theorem: If a Boolean algebra $\mathfrak{U}$ is $\mathfrak{m}^{\prime}$-complete for every infinite cardinal $\mathfrak{m}^{\prime}<\mathfrak{m}, A, A_{t} \in \mathfrak{U}$, $A=\bigcup_{i \in T} A_{t}$ and $\overline{\bar{T}} \leqslant \mathfrak{m}$, then there exist elements $B_{t} \in \mathfrak{U} \quad(t \in T)$ such that

$$
B_{t} \subset A_{t}, \quad B_{t} \frown B_{t^{\prime}}=0 \text { for } \quad t \neq t^{\prime} \text { and } A=\bigcup_{t \in T} B_{t} \text {. }
$$

Problem 1. Is this theorem true without the hypothesis that $\mathfrak{U}$ is $\mathfrak{m}^{\prime}$-complete for every $\mathfrak{m}^{\prime}<\mathfrak{m}$ ? (P 434).

Another problem of this kind is

Problem 2. Find, for every uncountable cardinal in, a Boolean m-algebra $\mathfrak{U}$ with the property: if the join $\bigcup_{t \in T} A_{t}$ exists in $\mathfrak{U}$ and $\overline{\bar{T}} \leqslant \mathfrak{m}$, then there exists a finite subset $T^{\prime} \subset T$ such that $\bigcup_{t \in T} A_{t}=\bigcup_{t \in T^{\prime}} A_{t}$. (P 435).

For $\mathfrak{m}=\boldsymbol{x}_{0}$ an example of such a Boolean algebra was given by Sierpiński [4].

Problems 3-6 which follow are connected with a classification of Boolean algebras discussed in my paper [7].

Problem 3. Find an example (for every uncountable cardinal m) of a weakly $\mathfrak{m}$-distributive Boolean $\mathfrak{m}$-algebra which is not $\mathfrak{m}$-distributive (P 436).

In the case where $\mathfrak{m}=\boldsymbol{x}_{0}$ such an example is given by non-atomic measure algebras (i. e. Boolean $\sigma$-algebras with a strictly positive finite o-measure). Other examples can be obtained e. g. by forming the direct 
union or the Boolean product of a non-atomic measure algebra and a $\sigma$-distributive $\sigma$-algebra. Thus the following problem arises:

Problem 4. Construct an example of a non- $\sigma$-distributive but weakly $\sigma$-distributive Boolean $\sigma$-algebra without using measure algebras (P 437).

Let $\mathcal{G}$ be a set of $\mathfrak{m}$-generators of a Boolean $\mathfrak{n}$-algebra $\mathfrak{U}$, and let $f$ be a mapping from $\mathfrak{S}$ into a Boolean $m$-algebra $\mathfrak{Z}$. If $f$ can be extended to an $\mathfrak{m}$-homomorphism $h$ from $\mathfrak{U}$ into $\mathfrak{B}$, then $f$ satisfies the following condition:

(a) if $\cap \bigcap_{t \in T}^{2 t} \eta(t) \cdot A_{t}=0$, where $\eta(t)= \pm 1, \quad A_{t} \in \odot$ and $T \leq \mathrm{m}$, then $\bigcap_{t \in T}^{g} \eta(t) \cdot f\left(A_{t}\right)=0$

Here the convention

$+1 \cdot A=A$ and $-1 \cdot A=-A=$ the complement of $A$

is assumed.

The necessary condition (a) for $f$ to have an extension to an m-homomorphism is not sufficient, in general. A Boolean $m$-algebra $\mathfrak{Z}$ is said to have the strong m-extension property if every mapping $f$ from a set $\mathfrak{S}$ of $\mathfrak{m}$-generators of an $\mathfrak{m}$-algebra $\mathfrak{U}$ into $\mathfrak{Z}$, such that (a) holds, cain be extended to an $\mathfrak{m}$-homomorphism from $\mathfrak{U}$ into $\mathfrak{Z}$. It is known (see Sikorski [6], theorem 34,1 ) that every m-field of sets has the strong m-extension property. Hence it follows easily that every $\mathfrak{m}$-distributive $\mathfrak{n}$-algebra has the strong nt-extension property (Sikorski [8], Sikorski and Traczyk [10]).

Problem 5. Is every m-algebra with the strong m-extension property necessarily m-distributive ? (P 438)

Consider now the particular case where the set $\mathbb{S}$ of m-generators of $\mathfrak{U}$ is a subalgebra of $\mathfrak{U}$. Then condition (a) implies that $f$ is a homomorphism. Clearly a homomorphism $f$ from $\mathfrak{S}$ into $\mathfrak{Z}$ satisfies condition (a) if and only if it satisfies the following condition:

(a') if $\bigcap_{t \in T}^{\mathfrak{T}}, A_{t}=0$ where $A_{t} \in \mathcal{S}$ and $\overline{\bar{T}}<\mathfrak{m}$, then $\bigcap_{t \in T}^{\mathfrak{B}} f\left(A_{t}\right)=0$.

Condition $\left(a^{\prime}\right)$ is necessary for the existence of an m-homomorphism from $\mathfrak{U}$ into $\mathfrak{Z}$ which is an extension of $f$. However, it is not sufficient. A Boolean $\mathfrak{m}$-algebra $\mathfrak{B}$ is said to have the weak m-extension property if every homomorphism $f$ from a subalgebra $\mathcal{S}$ of a Boolean m-algebra $\mathscr{U}$ into $\mathfrak{Z}$, such that

(i) $\mathfrak{S}$ m-generates $\mathfrak{U}$

(ii) $f$ satisfies $\left(a^{\prime}\right)$,

can be extended to an $\mathfrak{m}$-homomorphism from $\mathfrak{U}$ into $\mathfrak{Z}$

Clearly the strong m-extension property implies the weak m-extension property (therefore every m-distributive m-algebra has the weak m-extension property) but they are not equivalent. Dubins [1] proved that every measure algebra has the weak $\boldsymbol{\aleph}_{0}$-extension property. Matthes [2] proved a more general theorem: every weakly m-distributive Boolean m-algebra has the weak m-extension property.

Problem 6. Is every Boolean m-algebra with the weak m-extension property necessarily weakly m-distributive? (P 439).

It is known that it is always m-representable (see Sikorski [9]).

Pierce [3] proved that the $\mathfrak{m}$-completion of any $\mathfrak{m}$-distributive Boolean algebra is m-distributive. We mention here the analogous problem of Traczyk [11]:

Problem 7. Is the m-completion of a weakly m-distributive Boolean algebra also weakly m-distributive? (P 433)

Traczyk [11] proved that the answer is affirmative if the algebra in question satisfies the $\mathfrak{m}$-chain condition.

A pair $(i, \mathfrak{Z})$ is said to be an m-extension of a Boolean algebra 2 provided

$\left(e_{1}\right) \mathfrak{Z}$ is a Boolean $m$-algebra,

$\left(\mathrm{e}_{2}\right) i$ is an $\mathfrak{m}$-isomorphism from $\mathfrak{U}$ into $\mathfrak{Z}$,

$\left(\mathrm{e}_{3}\right) i(\mathfrak{U})$ m-generates $\mathfrak{Z}$

If $\left(i^{\prime}, \mathfrak{Z}^{\prime}\right)$ is another m-extension of $\mathfrak{U}$, we write

$(*)$

$$
(i, \mathfrak{Z}) \leqslant\left(i^{\prime}, \mathfrak{Z}^{\prime}\right)
$$

if there exists an $\mathfrak{m}$-homomorphism $h$ from $\mathfrak{B}^{\prime}$ onto (= into) $\mathfrak{B}$ such that $i=h i^{\prime}$.

Problem 8. Suppose $(i, \mathfrak{Z})$ is an m-completion of $\mathfrak{A}$. Does inequality (*) hold for every $\mathfrak{m}$-extension $\left(i^{\prime}, \mathfrak{Z}^{\prime}\right)$ of $\mathfrak{U}$ ? (P 440).

This problem was published by me for $\mathfrak{m}=\boldsymbol{\aleph}_{0}$ in a purely topological formulation in Colloquium Mathematicum 2 (1951), p. 151, P 77.

We know only that if $(i, \mathfrak{Z})$ is an m-completion of $\mathfrak{A}$, then $\left(i^{\prime}, \mathfrak{Z}^{\prime}\right) \leqslant$ $(i, \mathfrak{Z})$ never holds except in the trivial case where $\left(i^{\prime}, \mathfrak{Z}^{\prime}\right)$ is isomorphic to $(i, \mathfrak{Z})$ (i.e. there exists an isomorphism $h$ from $\mathfrak{B}^{\prime}$ onto $\mathfrak{Z}$ such that $i=h i^{\prime}$ ).

The affirmative solution of Problem 8 will solve automatically a few similar problems concerning minimal products of Boolean algebras (for details, see Sikorski [8] and Sikorski [9]).

Let $\mathfrak{m}$ and $\mathfrak{n}$ be infinite cardinals, $\mathfrak{n} \leqslant \mathfrak{m}$.

A pair $\left(\left(i_{t}\right)_{t \in T}, \mathfrak{Z}\right)$ is said to be a Boolean $(\mathfrak{m}, \mathfrak{n})$-product of an indexed set $\left(\mathfrak{U}_{t}\right)_{t \in T}$ of non-degenerate Boolean algebras provided

$\left(p_{1}\right) \mathfrak{Z}$ is a Boolean $m$-algebra,

$\left(p_{2}\right) i_{t}$ is an m-isomorphism from $\mathfrak{U}_{t}$ into 2 ,

$\left(\mathrm{p}_{3}\right)$ the union of all the subalgebras $i_{t}\left(\mathfrak{C}_{t}\right)$ m-generates $\mathfrak{B}$,

$\left(p_{4}\right)$ the subalgebras $i_{t}\left(\mathfrak{U}_{t}\right)$ are $\mathfrak{n}$-independent, i. $\Theta$.

$$
\cap_{t \in T^{\prime}}^{\mathfrak{g}}, i_{t}\left(A_{t}\right) \neq 0 \quad \text { for } \quad A_{t} \neq 0, A_{t} \in \mathfrak{U}_{t}, \quad \overline{\overline{T^{\prime}}} \leqslant \mathfrak{n}, \quad T^{\prime} \subset T .
$$


An example of an $(\mathfrak{m}, \mathfrak{n})$-product can be constructed as follows:

Let $X_{t}$ be the Stone space of $\mathfrak{U}_{t}$, let $g_{t}$ be the Stone isomorphism of $\mathfrak{U}_{t}$ onto the field of all clopen subsets of $X_{t}$, and let $X$ be the Cartesian product of all the spaces $X_{t}$. For every $A \epsilon \mathfrak{U}_{t}$, let $g_{t}^{*}(A)=$ the set of all points in $X$ whose $t^{\text {th }}$ coordinate is in $g_{t}(A)$.

Let $\mathscr{F}$ be the smallest field (of subsets of $X$ ) containing all the intersections $\bigcap_{t \in T^{\prime}} g_{l}^{*}\left(A_{t}\right)$, where $A_{t} \in \mathfrak{A}_{t}$ and $T^{\prime} \subset T, \overline{\bar{T}}^{\prime} \leqslant \mathfrak{n}$. Finally, let $(i, \mathfrak{B})$ be any m-extension of the Boolean algebra $\mathscr{F}$. Then

$$
\left(\left(i g_{t}^{*}\right)_{t \in T}, \mathfrak{Z}\right)
$$

is an $(\mathfrak{m}, \mathfrak{n})$-product of $\left(\mathfrak{U}_{t}\right)_{t \in \mathfrak{T}}$.

Problem 9. Is every $(\mathfrak{m}, \mathfrak{n})$-produet of $\left(\mathfrak{U}_{t}\right)_{t \in T}$ of the form $(* *)$ ? (P 441)

I should like also to recall that my problem on principal ideals in the field of all subsets of a set (Sikorski [5], P 61) is not yet solved.

\section{REFERENOES}

[1] L. E. Dubins, Generalized random variables, Transactions of the American Mathematical Society 84 (1957), p. $273-309$.

[2] K. Matthes, Über die Ausdehnung von \$-Homomorphismen Boolesche? Algebren, Zeitschrift für Mathematik, Logik und Grundlagen der Mathematik 6 (1960). p. $97-105$; (II) 7 (1961), p. $16-19$.

[3] R. S. Pierce, Distributivity and the normal completion of Boolean algebras. Pacifio Journal of Mathematics 8 (1958), p. 113-140.

[4] W. Sierpiński, Sur les ensembles presque ontenus les uns dans les autres, Fundamenta Mathematicae 35 (1948), p. 141-150.

[5] R. Sikorski, On an unsolved problem from the theory of Boolean algebras, (olloquium Mathematicum 2 (1949), p. 27-29.

[6] - Boolean algebras, Berlin-Göttingen-Heidelberg 1960.

[7] - Representation and distributivity of Boolean algebras, Colloquium Mathematicum 8 (1961), p. I-13.

[8] - On extensions and products of Boolean algebras, Fundamenta Mathematicat 53 (1963), p. 99-116.

[9] - Boolean algebras, second edition, in print.

[10]- and T. Traczyk, On free products of $\mathrm{m}-$ distributive Boolean algebras, Colloquium Mathematicum 11 (1963), p. 13-16.

[11] T. Traczyk, Minimal extensions of meakly distributive Boolean algebras, ibidem 11 (1963), p. 17-24.

Resu par la Rédaction le 26.11. 1962

$\begin{array}{lll}\text { VOL. XI } & 1963 & \text { FASC. } 1\end{array}$

A REMARK ON ABSULUTE-VALUEL ALGEBRAS

$\mathrm{BY}$

B. GLEIGHGEWIGHT (WROCEAW)

An algebra $A$ over the real field $R$ is called absolute-oulued if it is a normed space under a multiplicative norm | | i. e. a norm satisfying, in addition to the usual requirements, the condition $|x y|=|x||y|$ for all $x, y \in A$ (see [1]).

An operation * defined on $A$ is called an involution if it satisfies the following conditions:

$$
\begin{gathered}
(\lambda x+\mu y)^{*}=\lambda x^{*}+\mu y^{*}, \\
x^{* *}=x, \quad x x^{*}=x^{*} x, \quad(x y)^{*}=y^{*} x^{*}, \quad\left|x^{*}\right|=|x|
\end{gathered}
$$

for any $\lambda, \mu \in R$ and $x, y \in A$ (see [4]).

We say that an involution is non-trivial if it is different from the identity operation.

In every absolute-valued algebra $A$ with an involution we can introduce a new multiplication by means of the formula

$$
x \circ y=x^{*} y \text {. }
$$

The algebra $\perp$ with this product will be denoted by $\mathscr{K}^{\prime}(A) . \mathscr{K}^{\prime}(A)$ remains an absolute-valued algebra. The algebra $\mathscr{K}(A)$ is called a cracovian algebra generated by $A$ or an algebra induced by involution (see [2], [3]).

THEOREM. If $A$ is an absolute-valued algebra with a non-trinial innolution, then there exists in. $\mathscr{K}(A)$ an element e such that

$$
x o n=|x|^{2} e
$$

for any $x \in \mathscr{K}(A)$.

Proof. Using the well-known process of embedding linear normed spaces in Banach spaces, we can prove that the algebra $A$ can be extended to a complete algebra. Thus, without loss of generality, we may assume that the algebra $A$ is complete. For complete algebras it was proved in [4] that each element $x \in A$ can be represented as a sum $x=$ $=x_{1}+x_{2}$, where the elements $x_{1}$ and $x_{2}$ are self-adjoint and skew respec- 